Review Paper

\title{
LE MOTIF DU PONT DANS LA POÉSIE FRANÇAISE (HUGO, RIMBAUD, APOLLINAIRE)
}

UDC 821.133.1.09-1

\begin{abstract}
Nikola Bjelić
Université de Niš, Faculté de philosophie, Département de langue et littérature françaises, Niš, Serbie

Résumé. Vu que le motif du pont est associé à la ville comme sujet et objet de la poésie moderne, comme lieu d'errances poétiques, d'amour, d'inspiration, lieu de naissance du mal ou d'incompréhension du poète, ce qui peut se voir le mieux dans la deuxième partie des Fleurs du mal de Baudelaire (1857) intitulée "Tableaux parisiens », il n'est pas étonnant que motif soit présent dans la poésie à l'époque moderne, du romantisme jusqu'à nos jours. À cause de cela, notre recherche sur la présence et le rôle du pont dans la poésie française se base sur quelques poèmes des poètes les plus importants du $X I X^{e}$ et du XX $X^{e}$ siècles. L'objectif de notre analyse est le poème "Le Pont » du recueil Les Contemplations (1856) de Victor Hugo et le poème "Les Ponts» du recueil Illuminations (1895) d'Arthur Rimbaud. La recherche est complétée par l'analyse de l'un des plus beaux poèmes français du XX $X^{e}$ siècle, "Le Pont Mirabeau » du recueil Alcools (1913) de Guillaume Apollinaire.
\end{abstract}

Mots-clés : poésie, pont, ville, romantisme, symbolisme, amour

\section{INTRODUCTION}

Il n'est pas possible, nous semble-t-il, de parler des ponts, de leur signification et leur importance, sans mentionner au début le célèbre texte d'Ivo Andrić sur les ponts, dans lequel le grand écrivain écrit que, «de tout ce que l'homme dans son élan vital élève ou construit, il n'est rien [...] de mieux ni de plus précieux que les ponts », qui à ses yeux « ont plus d'importance que les maisons, un caractère plus sacré, parce que plus commun à tous, que les temples », car «ils appartiennent à tout le monde, sont identiques et utiles à tout un chacun, ils sont toujours construits de manière réfléchie, à la croisée du plus

Submitted July 1, 2019; Accepted September 23, 2019

Corresponding author: Nikola Bjelić

University of Niš, Faculty of Philosophy

E-mail: nikola.bjelic@filfak.ni.ac.rs 
grand nombre des besoins humains, ils possèdent plus de permanence que toute autre construction et ne servent aucune fin qui soit secrète ou maligne » (Andrić 2009, 308). ${ }^{1}$ Les ponts subsistent donc depuis des siècles en tant que constructions les plus importantes, les plus utiles et les plus magnifiques de l'esprit humain.

Personne n'a écrit sur les ponts de manière aussi belle et précise que notre prix Nobel, révélant leur véritable sens, mais traitant également de leur fonction symbolique. Dans $L e$ Dictionnaire des symboles, Jean Chevalier et Alain Gheerbrant notent que «le symbolisme du pont, permettant de passer d'une rive à l'autre, est l'un des plus universellement répandus $(1982,777)$. Ce passage d'une rive à l'autre, hormis le sens littéral, peut avoir un certain nombre de sens figurés. C'est aussi «le symbole de la traversée d'une eau, par exemple celle qui sépare la vie terrestre de la vie extraterrestre » (Biderman 2004, 242).

Le motif du pont dans la littérature française, et surtout dans la poésie, est lié à la ville en tant que sujet et objet de la poésie moderne. La ville est un lieu d'errance poétique, d'amour, d'inspiration, lieu de naissance du mal ou d'incompréhension du poète. La ville est créée toujours autour des fleuves ou des rivières qui sont les sources de vie. Devant les rivières, l'homme est toujours provoqué par le besoin de les retenir, de les vaincre, afin de réaliser le rêve d'atteindre l'autre rive, c'est-à-dire l'inconnu, l'extraterrestre. C'est pourquoi il n'est pas étonnant que la ville ne soit présente de manière significative dans la poésie française qu'à l'époque moderne, depuis l'avènement du romantisme, de Victor Hugo et Baudelaire jusqu'aux poètes contemporains. Elle a également pris une place importante dans la poésie du premier poète moderne d'Europe, François Villon, qui a profondément influencé la poésie française moderne. C'est pourquoi, dans notre travail, nous nous limitons à l'exemple des trois poètes : Victor Hugo, (1802-1885), Arthur Rimbaud (1854-1891) et Guillaume Apollinaire (1880-1918), en tant que poètes magistraux de la poésie française du $\mathrm{XIX}^{\mathrm{e}}$ et du $\mathrm{XX}^{\mathrm{e}}$ siècles.

\section{2. « LE PONT » DE VICTOR HUGO}

Dans l'immense œuvre poétique du coryphée de la poésie française Victor Hugo, le poème « Le Pont » occupe une place significative.

\section{LE PONT}

J'avais devant les yeux les ténèbres. L'abîme

Qui n'a pas de rivage et qui n'a pas de cime

Était là, morne, immense ; et rien n'y remuait.

Je me sentais perdu dans l'infini muet.

Au fond, à travers l'ombre, impénétrable voile,

On apercevait Dieu comme une sombre étoile.

Je m'écriai : - Mon âme, ô mon âme ! il faudrait,

Pour traverser ce gouffre où nul bord n'apparaît,

Et pour qu'en cette nuit jusqu'à ton Dieu tu marches,

\footnotetext{
${ }^{1}$ „Od svega što čovek u životnom nagonu podiže i gradi, ništa nije u mojim očima bolje i vrednije od mostova. Oni su važniji od kuća, svetiji od hramova. Svačiji i prema svakom jednaki, korisni, podignuti uvek smisleno, na mestu na kom se ukrštava najveći broj ljudskih potreba, istrajniji su od drugih građevina i ne služe ničem što je tajno ili zlo“. (Andrić 1976, 199)
} 
Bâtir un pont géant sur des millions d'arches.

Qui le pourra jamais? Personne! Ô deuil! Effroi!

Pleure! - Un fantôme blanc se dressa devant moi

Pendant que je jetai sur l'ombre un xil d'alarme,

Et ce fantôme avait la forme d'une larme ;

$C$ 'était un front de vierge avec des mains d'enfant;

Il ressemblait au lys que sa blancheur défend;

Ses mains en se joignant faisaient de la lumière.

Il me montra l'abîme où va toute poussière,

Si profond que jamais un écho n'y répond,

Et me dit : -Si tu veux, je bâtirai le pont.

Vers ce pâle inconnu je levai ma paupière.

- Quel est ton nom? lui dis-je. Il me dit :-La prière.

Jersey, décembre 1852. (Hugo 1856b, 187-188)

Ce poème, comme c'est indiqué à la fin, a été créé en décembre 1852, sur l'île de Jersey, située dans La Manche, entre la France et la Grande-Bretagne. Hugo était en exil volontaire sur cette île appartenant au Royaume-Uni de 1852 à 1855 , puis il a déménagé sur l'île de Guernesey. Il est parti en exil après un coup d'État qui a été effectué le 2 décembre 1851 par l'unique président de la Deuxième République française Louis-Napoléon Bonaparte, qui a décidé de restaurer l'Empire en se proclamant l'empereur des Français. Profondément opposé à cela, bien qu'en 1848, lors de la première élection présidentielle, il ait soutenu la candidature de Louis-Napoléon et non son collègue, le poète Alfonse de Lamartine, Hugo écrit et publie en 1852 un pamphlet politique contre Louis-Napoléon, intitulé Napoléon le Petit, raison pour laquelle il sera obligé de quitter l'année suivante la France, où il ne reviendra qu'après 18 ans d'exil, en septembre 1870 (Winok 2018, 129-145).

Pendant son exil sur les îles Anglo-Normandes, persécuté par le souvenir de sa fille tragiquement décédée, Hugo se consacre au spiritisme. Deuxième de ses cinq enfants, sa fille préférée Léopoldine, avec son mari, Charles Vacquerie, s'est noyée dans la Seine à Villequier le 4 septembre 1843, huit jours après son dix-neuvième anniversaire. Cet événement, le plus tragique dans la longue vie du grand écrivain, qu'il n'a appris que quatre jours plus tard dans la presse, va l'influencer de manière décisive, si bien qu'il cessera de publier des œuvres littéraires pendant dix ans. Mais la mort de Léopoldine inspirera certains des plus beaux poèmes de la poésie française, rassemblés dans son recueil le plus important, Les Contemplations (1856). Ce recueil compte 158 poèmes qui ont été créés principalement entre 1843 et 1855, bien qu'il y en ait qui sont plus anciens. Ce recueil de poésie méditative est divisé en deux parties, chacune comprenant trois livres. Dans la «Préface» de la première édition de 1856, Hugo écrit que, dans Les Contemplations, «c'est une âme qui se raconte dans ces deux volumes : Autrefois, Aujourd'hui. Un abîme les sépare, le tombeau » (Hugo 1856a, III). La première partie, intitulée « Autrefois », contient trois livres dont le thème est le passé, la vie de 1830 à 1843 , c'est-à-dire avant l'événement tragique, et ce sont: 1. «Aurore », 2. «L'âme en fleur» et 3. «Les luttes et les rêves ». La deuxième partie, intitulée «Aujourd'hui », est composée de trois livres dont le thème est le présent, le temps après la mort tragique, et ce sont : 4. «Pauca Meae »(Versets pour ma fille), 5. «En marche » et 6 . «Au bord de l'infini». Ce dernier livre, «Au bord de l'infini», contient vingt-six poèmes qui représentent la méditation métaphysique du poète, grâce à laquelle, à travers des images d'anges, de visions, de fantômes, d'êtres surnaturels, il devient le premier parmi les poètes voyants, annonçant la poésie symboliste. Le poème « Le Pont » ouvre ce livre. 
«Le Pont » est constitué de vingt-deux alexandrins à rimes parallèles. Le poème peut être divisé en quatre parties. La première partie est formée des six premiers vers, dans lesquels est décrite l'angoisse du poète devant l'inconnu. Il se trouve, perdu, à un endroit sombre et incertain, devant un abîme sans fin, sans rivage, au fond duquel on peut sentir la présence de Dieu en tant que «sombre étoile ». Cet oxymore ferme cette partie et ouvre la deuxième qui contient les cinq vers suivants. C'est un appel au secours que le poète adresse à son âme, lui disant que, pour atteindre ce dieu, on devrait construire un pont gigantesque aux millions d'arches. Dans la troisième partie, composée de neuf vers, le poète décrit un fantôme blanc en forme de larme, pur et innocent, aux mains enfantines, illuminé par la lumière, qui apparaît lors de son appel au secours, lui disant qu'il accomplira son vœu de bâtir le pont entre Dieu et lui. La quatrième partie est constituée des deux derniers vers, dans lesquels le poète offre la conclusion, le dénouement, affirmant que la prière représente un moyen par lequel un pont invisible mais ferme peut être construit entre ce monde et le monde extraterrestre, entre le poète, un homme qui souffre, et un Être Suprême, Dieu, qui seul peut apporter le réconfort à son âme souffrante.

En analysant le lexique utilisé par le poète, on pourrait conclure que le poème est invisiblement divisé en deux parties égales en nombre de vers, dans lesquelles Hugo fait varier habilement son jeu romantique préféré du clair-obscur. Dans les onze premiers vers, Hugo utilise le champ lexical de l'obscurité (ténèbres, morne, ombre, nuit, deuil, effroi) et celui de la profondeur (abîme, gouffre), ce qui annonce que l'appel au secours, adressé dans cet endroit sombre et morne, est vain et absurde, car il n'y a pas d'espoir. Dans les onze autres vers, il utilise le champ lexical du clair (blanc, blancheur, lumière, pâle) et celui biblique (vierge, enfant, lys, abîme, poussière, écho, prière), ce qui montre un coup de théâtre, car il indique clairement un dénouement inattendu, surprenant et heureux, qui redonne espoir. Ce dénouement dramatique est pareil au miracle biblique, à la connaissance qui ouvre les yeux. L'apparition de la fleur biblique, le lys, rappelle le pouvoir de la beauté et de l'amour. La prière, la seule qui puisse relier les deux mondes, est décrite comme innocente, pure comme un enfant, compatissante comme des larmes, belle comme un lys, c'est une lumière qui redonne espoir à la vie.

De tout ce qu'on a dit, il est clair que le pont dont parle Hugo n'est ni un bâtiment physique ni une construction ; par conséquent, il ne peut non plus être relié au topos de la ville, car il s'agit d'une catégorie métaphysique. La prière est symboliquement décrite comme un pont, moyen de connexion et de l'alliance du clair et de l'obscur, de la lumière et des ténèbres, du terrestre et de l'extraterrestre, de l'intérieur et de l'extérieur. Chez Hugo, le pont représente en fait ce "passage [...] de la terre au ciel, de l'état humain aux états suprahumains, de la contingence à l'immortalité, du monde sensible au monde supra-sensible » (Chevalier, Gheerbrant 1982, 777), dont on parle dans Le Dictionnaire des symboles. Avec ce poème, Hugo a considérablement annoncé le symbolisme, il en est même le précurseur.

\section{3. «PONTS » DE RIMBAUD}

L'un des précurseurs du symbolisme, des pionniers de la poésie moderne et l'un des poètes qui ont apporté à la poésie des changements révolutionnaires fut Arthur Rimbaud. Mais, il était disciple d'un autre poète, poète le plus important à avoir influencé la naissance du symbolisme en France - Charles Baudelaire (1821-1867). Avec son recueil Fleurs du mal, publié en 1857, donc un an après Les Contemplations d'Hugo, Baudelaire apporte un changement radical à la poésie dans les décennies à venir. Avec lui, le topos de la ville 
occupe une place centrale dans la poésie. Paris, avec ses immeubles, ses places et ses ponts, avec ses prostituées et ses dandys, avec toutes ses beautés et ses laideurs, c'est-à-dire avec toutes ses oppositions, devient la scène, le lieu de l'errance poétique, de l'amour, de l'inspiration, le lieu de la naissance du mal ou de l'incompréhension du poète, ce qui est le plus visible dans la deuxième partie de son recueil, intitulée « Tableaux parisiens ».

Cependant, le pont en tant que motif n'est mentionné qu'une seule fois dans les Fleurs du mal, dans le deuxième vers de la troisième strophe de la deuxième partie du poème final « Voyage » :

Notre âme est un trois-mâts cherchant son Icarie ;

Une voix retentit sur le pont :, Ouvre l'œil! “

Une voix de la hune, ardente et folle, crie :

„Amour... gloire... bonheur! “Enfer! c'est un écueil!

(Baudelaire 1972, 124, mis en gras par nous)

Ici, le pont est un symbole du passage, du franchissement, car «les ponts servent non seulement à relier, mais, dans un sens symbolique, ils représentent aussi la situation du passage à une autre forme d'existence, qui ne peut être comprise en réflexion que par des rituels précisément déterminés du passage » (Biderman 2004, 242). Le pont ne représente pas seulement un passage, c'est aussi un voyage initiatique qui peut être souvent périlleux (Chevalier, Gheerbrant 1982, 777), car il est incertain. C'est justement de ce passage, qui va de la vie à la mort, que parle Baudelaire dans son poème, dans lequel la mort n'est pas perçue de manière négative et comme néant, mais comme une option, une possibilité. Elle est « l'Inconnu » dans lequel il faut «plonger » afin de « trouver du nouveau » (Baudelaire 1972, 128).

Le premier grand disciple de Baudelaire fut Arthur Rimbaud (Bonnefoy 1961, 43-49). L'idée centrale de Rimbaud était de «changer la vie» pour pouvoir transformer le monde. Conformément à sa propre idée, il quitte l'écriture et Paris à l'âge de 20 ans et part au monde pour vivre l'aventure poétique qu'il a d'abord décrite dans ses vers, en particulier dans le poème célèbre «Le Bateau ivre ». Ses poèmes sont restés derrière lui pour changer le monde poétique parisien, mais il ne s'en souciait plus.

Sous l'influence de Baudelaire, Rimbaud écrivait aussi des poèmes en prose, qui ont été publiés dans deux recueils : Une saison en enfer et Illuminations. Son poème « Ponts » est publié dans les Illuminations. Il a été créé en 1873 et publié dans la première édition des Illuminations en 1886 comme partie finale du poème «Ouvriers ». Dans les éditions suivantes, ce dernier paragraphe est séparé en un poème particulier, intitulé « Ponts » :

Des ciels gris de cristal. Un bizarre dessin de ponts, ceux-ci droits, ceux-là bombés, d'autres descendant ou obliquant en angles sur les premiers, et ces figures se renouvelant dans les autres circuits éclairés du canal, mais tous tellement longs et légers que les rives, chargées de dômes, s'abaissent et s'amoindrissent. Quelques-uns de ces ponts sont encore chargés de masures. D'autres soutiennent des mâts, des signaux, de frêles parapets. Des accords mineurs se croisent et filent, des cordes montent des berges. On distingue une veste rouge, peut-être d'autres costumes et des instruments de musique. Sont-ce des airs populaires, des bouts de concerts seigneuriaux, des restants d'hymnes publics? L'eau est grise et bleue, large comme un bras de mer. - Un rayon blanc, tombant du haut du ciel, anéantit cette comédie. (Rimbaud 1892,73) 
Après « Ponts » se trouve un des poèmes intitulés «Villes ». Le poème «Ponts » fait partie, ainsi que les «tableaux parisiens » de Baudelaire, des topö̈ de la ville, c'est là que repose leur modernité. Le poème de Rimbaud représente l'image impressionniste d'une métropole urbaine moderne (Bertolino 1991). L'analyse de nombreux détails a permis de conclure que la ville que le poète décrit est Londres, bien que certains critiques pensent qu'il s'agit plutôt d'autres villes telles que Venise, Bruxelles ou Amsterdam. Nous sommes plus près de croire que Rimbaud a pourtant peint ici la capitale britannique, car plusieurs faits le signalent. Premièrement, Rimbaud évoque des canaux, qui sont nombreux à Londres, ce qui est complété par l'image du ciel gris typique de cette ville. La mention du dôme réfère clairement à un grand nombre de dômes d'église qui existaient sur la Tamise au XVIII ${ }^{\mathrm{e}}$ siècle, qui ont été démolis mais conservés dans les gravures. L'un d'eux, le dôme de la cathédrale Saint-Paul, domine toujours le panorama de Londres. En outre, il est bien connu qu'à l'époque de la création de ce poème, Rimbaud séjournait à Londres avec Verlaine, de septembre 1872 à juillet 1873, une des périodes les plus célèbres et les plus bouleversantes de leurs vies.

Le poème de Rimbaud est avant tout un tableau inspiré des ponts concrets, existants, et du paysage urbain. Mais le poète transforme cette réalité en une image suggestive qui échappe à cette réalité, devenant une image abstraite proche des toiles des impressionnistes et des cubistes (bien que le mouvement cubiste ne naisse qu'au $\mathrm{XX}^{\mathrm{e}}$ siècle), ce qui souligne la nécessité d'une relation entre ce qui est vu et ce qui est vécu (ressenti), ouvrant la voie de la poésie moderne qui s'appuie de plus en plus sur les arts visuels (cf. Popović 2016, 3757). Le champ lexical utilisé par le poète fait référence aux beaux-arts. Le premier mot, ciels, est utilisé sous la forme du pluriel qu'on utilise principalement lorsqu'il s'agit de la peinture (des ciels, au lieu de l'habituel des cieux), en lien avec les mots dessin et figures. Le poète utilise une gamme complète de couleurs dans laquelle la place dominante est occupée par les couleurs pâles (gris de cristal, l'eau grise et bleue et blanc), le rayon (rayon) et les couleurs vives (rouge), qui soulignent d'autres couleurs. Les lignes nettes qu'on pressent (droits, bombés, descendant, longs, cordes, dômes, circuits, angles) nous rappellent la peinture cubiste. Au tout début, le poète dit que la scène qu'il observe est « un dessin bizarre ». Ce dessin est plein de lignes horizontales (ponts, canal, rives, parapets) et verticales (mâts, cordes), ce qui se réfêre à l'art graphique.

Au début, cette image est statique, mais elle commence à vivre peu à peu, autrement dit elle devient «vivante (non-statique) reflétant la réalité matérielle » (Popović 2016, 34). Ce qui contribue à cela, ce sont les sons (accords mineurs, instruments de musique, airs populaires, concerts seigneuriaux, hymnes publics), un grand nombre d'allitérations, d'assonances et de rimes intérieures cachées, ainsi que l'utilisation des verbes d'action (s'abaissent, s'amoindrissent, croisent, filent, montent). «Une veste rouge » et «d'autres costumes » font référence au théâtre et aux fêtes sur les places, ce qui donne une grande vivacité au tableau. À la fin, le poète lui-même utilise le mot comédie, car la scène qu'il observe, peut-être de la rivière ou du bateau, est en mouvement. La dernière phrase, «Un rayon blanc, tombant du haut du ciel, anéantit cette comédie », est séparée du texte précédent par un tiret, comme si le poète voulait même visuellement décrire une rupture soudaine, une fin inattendue. Le rayon qui tombe de la hauteur céleste indique une intervention divine possible, qui arrête ce jeu féerique joyeux, ce qui introduit une ligne sombre dans le poème. Ainsi, le poème «Ponts » pourrait être interprété comme une allégorie de la grave condition humaine. Même si la description montre que le pont est une construction solide, elle est toujours frêle et fragile, car un seul rayon suffit à la détruire. Ce rayon blanc est la lumière 
qui met fin à une rêverie et à une extase dans lesquelles l'homme se sentait heureux. Sans ce rayon, sans cette lumière, tout n'était qu'un rêve ou une illusion éphémère, le fruit de l'imagination ou une comédie qui s'effondre toute seule. Cette chute à la fin fait référence à la chute d'Icare, ce qui montre également le déclin d'un rêve. La fin pessimiste annonce les dangers guettant tous ceux qui oseraient essayer de changer l'ordre de la nature.

Une telle fin exprime aussi, ironiquement, la fragilité de toute création artistique. L'œuvre d'art est toujours une «illumination», excitante, mais périssable. Les ponts sont des symboles qui expriment l'unité du monde, terrestre et extraterrestre. Ils relient les rives opposées, mais, bâtis entre le ciel et la terre, ils relient le terrestre et l'extraterrestre.

\section{4. «LE PONT MirabEAU » D’APOLlinaire}

Le plus célèbre de tous les poèmes français qui chantent les ponts, l'un des plus analysés (Bjelić 2002) est sans aucun doute «Le Pont Mirabeau» de Guillaume Apollinaire. Il a d'abord été publié dans la revue Les soirées de Paris en février 1912, puis réimprimé dans le recueil Alcools en 1913 (Apollinaire 1965, 47). Il s'agit d'une élégie dont les thèmes principaux sont l'amour, la fuite du temps et la mémoire.

\section{LE PONT MIRABEAU}

Sous le pont Mirabeau coule la Seine

$$
\text { Et nos amours }
$$

Faut-il qu'il m'en souvienne

La joie venait toujours après la peine

Vienne la nuit sonne l'heure

Les jours s'en vont je demeure

Les mains dans les mains restons face à face

Tandis que sous

Le pont de nos bras passe

Des éternels regards l'onde si lasse

Vienne la nuit sonne l'heure

Les jours s'en vont je demeure

L'amour s'en va comme cette eau courante L'amour s'en va

Comme la vie est lente

Et comme l'Espérance est violente

Vienne la nuit sonne l'heure

Les jours s'en vont je demeure

Passent les jours et passent les semaines

$$
\text { Ni temps passé }
$$

Ni les amours reviennent

Sous le pont Mirabeau coule la Seine

Vienne la nuit sonne l'heure

Les jours s'en vont je demeure 
Le poème se compose de quatre quatrains et quatre distiques comme refrains. Là, le poète évoque son amour pour l'artiste-peintre Marie Laurencin. Il est toujours rentré de chez elle au quartier d'Auteuil, où il habitait, en traversant le célèbre pont qui est situé à la partie ouest de Paris (Apollinaire 1965, 47). L'amour fini, le poète se trouve sur le pont et fait appel à la mémoire des beaux moments. Il conclue que tout passe, comme la Seine qui coule au-dessous, et la seule chose qui est permanente est la mémoire ressuscitée par le pont. Cependant, elle change aussi avec le temps, comme la Seine change sans cesse. Ici, le pont joue le même rôle que la célèbre petite madeleine de Proust, le rôle de résurrection de la mémoire involontaire, car il y réveille toujours les souvenirs des jours heureux. L'image de la deuxième strophe est significative. Là, le poète évoque un couple amoureux qui se tient face à face «les mains dans les mains». La position de leurs corps ressemble à la structure du pont, symbolisant sa persistance, et le verbe à l'impératif (restons) indique le désir du poète de maintenir leur liaison physique et de la rendre stable.

Par sa forme (Bjelić 2002, 597-600), le poème lui-même nous fait penser à l'image du pont, car les strophes nous associent au pont, tandis que les refrains seraient les arcs et les piliers sous lesquels coule la vie. Cette image suggestive rend ce poème d'Apollinaire proche à ses calligrammes, forme «intrigante qui appartient d'une part à l'esthétique des arts visuels, tandis que, par son contenu verbal, elle appartient à l'art poétique, ce qui la classe, après tout, dans des formes littéraires » (Popović 2016, 14).

Comme chez Rimbaud, le pont est ici aussi un symbole qui exprime l'unité du monde, terrestre et extraterrestre.

\section{EN GUISE DE CONCLUSION}

Les trois poèmes analysés nous renvoient chacun à nouveau, de manière étrange et cyclique, à la pensée célèbre d'Ivo Andrić selon laquelle les ponts sont les bâtiments les plus importants et les plus sacrés, dont le rôle est de surmonter, de franchir, de lier et de relier deux rives ou deux mondes différents. À la fin de son poème en prose (car nous lisons ce texte de telle manière), Andrić conclut que

tous les faits parlants de notre existence - les pensées, les efforts, les regards, les sourires, les paroles, les soupirs - tout aspire à gagner l'autre rive, c'est une sorte d'objectif à atteindre et qui ne prend son sens véritable qu'une fois atteint. Tout tient d'un obstacle à surmonter et à franchir : le désordre, la mort, l'absurdité. Car tout n'est qu'un passage, un pont dont les extrémités se perdent dans l'infini et par rapport auquel tous les ponts terrestres ne sont que des jeux puérils, de pâles symboles. Alors que tous les espoirs que nous formons nous viennent de l'autre côté. (Andrić 2009, 310) ${ }^{2}$

Note : Cet article est rédigé dans le cadre du projet scientifique Les langues, les littératures et les cultures romanes et slaves en contact et en divergence, $N^{o}$ 81/1-17-8-01, approuvé par la Faculté de Philosophie de l'Université de Niš et soutenu par l'Agence universitaire de la Francophonie.

\footnotetext{
${ }^{2}$ „sve čim se ovaj naš život kazuje - misli, napori, pogledi, osmesi, reči, uzdasi - sve to teži ka drugoj obali, kojoj se upravlja kao cilju, i na kojoj tek dobiva svoj pravi smisao. Sve to ima nešto da savlada i premosti: nered, smrt ili nesmisao. Jer, sve je prelaz, most čiji se krajevi gube u beskonačnosti, a prema kom su svi zemni mostovi samo dečije igračke, bledi simboli. A sva je naša nada s one strane.“(Andrić 1976, 201)
} 
Une partie de cette recherche a été représentée au Colloque scientifique international "Le pont dans la littérature, l'histoire et la cinématographie » qui s'est déroulé à l'Institut Andrić à Andrićgrad (Višegrad, Bosnie-Herzégovine) les 28-30 octobre 2016.

\section{RÉFÉRENCES BIBLIOGRAPHIQUES}

Andrić, Ivo. « Mostovi », dans Staze, lica, predeli. Sarajevo, Zagreb, Beograd, Ljubljana, Skopje, Svjetlost, Mladost, Prosveta, Državna založba Slovenije, Misla, 1976, p. 199-201.

Andrić, Ivo. « Les Ponts », dans Europe. N ${ }^{\circ}$ 960. Paris. Traduit par Alain Cappon. 2009, p. 308-310. https://serbica.ubordeaux-montaigne.fr/index.php/p/399-ponts-les-mostovi-ivo-andri

Apollinaire, Guillaume. Alcools. Paris, Librairie Larousse, 1965.

Baudelaire, Charles. Fleurs du mal. Paris, Le livre de poche, 1972.

Bertolino, Nikola. Fenomen Rembo. Beograd, Nolit, 1991.

Bonnefoy, Yves. Rimbaud. Paris, Seuil, 1961.

Biderman, Hans. Rečnik simbola. Beograd, Plato, 2004.

Bjelić, Nikola. «O prevođenju neosimbolističke poezije na primerima Pasternaka i Apolinera », Prevodna književnost. Beograd, UKPS, 2002, 595-603.

Chevalier, Jean et Gheerbrant, Alain. Le Dictionnaire des symboles. Paris, Robert Laffont, 1982.

Hugo, Victor. Les Contemplations I: Autrefois - 1830-1843. Paris, Michel Lévy frères, 1856a. http://gallica. bnf.fr/ark:/12148/bpt6k6102854c.

Hugo, Victor. Les Contemplations II: Aujourd'hui - 1843-1856. Paris, Michel Lévy frères, 1856b. http://gallica. bnf.fr/ark:/12148/bpt6k6105948x.

Popović, Diana. Poetika Apolinerovih kaligrama. Sremski Karlovci-NoviSad, Izdavačka knjižarnica Zorana Stojanovića, 2016.

Rimbaud, Arthur. Les Illuminations. Une Saison en enfer. Paris, Léon Vanier, 1892. http://gallica.bnf.fr/ark:/ 12148/btv1b8618404b?rk=85837;2.

Winok, Michel. Le monde selon Victor Hugo. Paris, Tallandier, 2018. https://www.tallandier.com/livre/lemonde-selon-victor-hugo/

\section{MOTIV MOSTA U FRANCUSKOJ POEZIJI (IGO, REMBO, APOLINER)}

Imajući $u$ vidu da se motiv mosta vezuje za grad kao subjekat $i$ objekat moderne poezije, kao mesto pesničkih lutanja, ljubavi, inspiracije, mesto rađanja zla ili nerazumevanja pesnika, što je najbolje vidljivo u drugom delu Bodlerovog Cveća zla (Fleurs du mal, 1857) naslovljenom „Pariske slike“, nije ni čudno što je on značajnije prisutan u poeziji tek u moderno doba, od pojave romantizma naovamo, počev od Viktora Igoa preko Bodlera, pasve do savremenih pesnika. Zbog toga se naše istraživanje o prisustvu i ulozi mosta u francuskoj poeziji bazira na nekoliko pesama najznačajnijih pesnika XIX i početka XX veka. Predmet našeg istraživanja čine pesme „Most" ("Le Pont ») iz zbirke Kontemplacije (Contemplations, 1856) Viktora Igoa i „Mostovi“ ("Les Ponts ») iz zbirke Iluminacije (Illuminations, 1895) Artura Remboa. Istraživanje je upotpunjeno analizom jedne od najlepših francuskih pesama XX veka, , Most Mirabo “ ( L Le Pont Mirabeau ») iz zbrke Alkoholi (Alcools, 1913) Gijoma Apolinera.

Ključne reči: poezija, most, grad, romantizam, simbolizam, ljubav. 